The Department of Computer Sciences at Purdue University generously contributed time on its new computer installation for this project. My thanks go especially to the director, Dr. Conte.

Purdue University

West Lafayette, Indiana

1. L. E. Dickson, "Proof of the ideal Waring theorem for exponents 7-180," Amer.J. Math., v. 58,1936, p. $521-529$.

2. — "Solution of Waring's problem," Amer. J. Math., v. 58, 1936, p. 530-535.

3. " "The Waring problem and its generalizations," Bull. Amer. Math. Soc., v. 42. 1936, p. 833-842.

4. K. MAHLER, "On the fractional parts of the powers of a rational number (II)," Mathematika, v. 4, 1957, p. 122-124.

5. Hans-Heinrich Ostmann, Additive Zahlentheorie, zweiter Teil, Springer-Verlag, 1956, p. $81-82$.

6. S. S. PIllaI, "On Waring's problem," Indian J. Math. n.s., v. 2, 1936, p. 16-44.

7. D. RIDour, "Rational approximations to algebraic numbers," Mathematika, v. 4, 1957, p. $125-131$.

\title{
Fermat Numbers and Mersenne Numbers
}

\section{By J. L. Selfridge and Alexander Hurwitz}

An IBM 7090 computer program, and results of testing Mersenne numbers $M_{p}=2^{p}-1$ with $p$ prime, $p<5000$, have been described by Hurwitz [1]. This paper describes modifications made to his program, and further computational results. The main results are that the Fermat number $F_{14}$ is composite and that $2^{p}-1$ is composite if $5000<p<6000$.

The computer program, originally written with the idea of testing $2^{n}-1$ for $n=M_{13}$, soon showed that the machine makes occasional errors. At least four machine errors occurred during runs on this number before two results agreed. Due partly to the immediate availability of standby time, the program was then launched in the region $3300<p<5000$.

When this work was nearly complete, the routine was modified to incorporate a check modulo $2^{35}-1$ after each squaring and another after each reduction modulo $2^{p}-1$. These checks enabled the routine to recover and proceed automatically after a machine error. A message was printed that a squaring (or reduction) error had occurred. In fact, this happened several times.

Another modification enabled the program to compute $3^{2^{n}}$ modulo the Fermat number $F_{m}=2^{2^{m}}+1$. When $n=2^{m}-1$ the residue was output, with a result congruent to -1 if and only if $F_{m}$ is prime.

After testing the program using $F_{10}$ (see Robinson [5]), we proceeded to test $F_{14}$. The computation was divided into 64 parts, and the results of the first 25 of these were checked against those of Paxson [3], who very kindly sent us copies of his intermediate residues. The rest of the computation was done twice, with complete agreement. We have also checked the final residue obtained by Paxson [3] in the testing of $F_{13}$. The result that $F_{14}$ is composite was announced in [2].

Received August 12, 1963. The preparation of this paper was sponsored by the Office of Naval Research. 
FERMAT NUMBERS AND MERSENNE NUMBERS

TABLE 1

\begin{tabular}{r|r|c|c|c}
\hline$m$ & $n$ & $R \bmod 2^{36}$ & $R \bmod 2^{36}-1$ & $R \bmod 2^{35}-1$ \\
\cline { 2 - 3 } \cline { 5 - 5 } & 127 & 035100542404 & 514165207640 & 053153335617 \\
8 & 255 & 531023263263 & 407614543114 & 344141643032 \\
13 & 8191 & 607301005536 & 611677367012 & 031455470517 \\
14 & 16383 & 622476273512 & 016631677043 & 161031465216 \\
17 & 20 & 176536764625 & 415751561367 & 155276133751 \\
\hline
\end{tabular}

TABLE 2

\begin{tabular}{c|c||c|c||c|c}
\hline$p$ & $R$ & $p$ & $R$ & $p$ & $R$ \\
\hline 5023 & 35472 & 5479 & 17227 & 5783 & 15446 \\
5077 & 27063 & 5503 & 26142 & 5813 & 25753 \\
5081 & 74607 & 5527 & 41614 & 5839 & 24031 \\
5099 & 67662 & 5573 & 34740 & 5851 & 37460 \\
5113 & 20010 & 5581 & 31446 & 5857 & 11252 \\
5153 & 52273 & 5591 & 52563 & 5869 & 00764 \\
5309 & 40357 & 5641 & 21342 & 5879 & 52670 \\
5333 & 44244 & 5647 & 40775 & 5897 & 30763 \\
5351 & 05171 & 5653 & 50244 & 5923 & 16616 \\
5387 & 54357 & 5669 & 57031 & 5953 & 32461 \\
& & & & \\
5407 & 51133 & 5689 & 32731 & 5987 & 66731 \\
5419 & 70701 & 5693 & 47014 & & \\
5443 & 51737 & 5701 & 33577 & & \\
5471 & 52563 & 5737 & 07151 & & \\
\hline
\end{tabular}

In addition, as a debugging aid for those who wish to test $F_{17}$, we computed $3^{2^{20}}$ modulo $F_{17}$. The complete testing of $F_{17}$ would take 128 full weeks of machine time on the IBM 7090. It seems much more economical to search for small factors of $F_{17}$ than to perform this test.

The final residues in the testing of $F_{7}, F_{8}, F_{13}$ and $F_{14}$, and the residue of $3^{220}$ $\left(\bmod F_{17}\right)$, have been put on punched cards, together with check sums. A summary of these residues is given in Table 1, and copies of the cards are available for checking purposes. The seven intermediate residues of $3^{2^{n}}\left(\bmod F_{14}\right)$ where $n \equiv 0(\bmod$ 2048), and the complete values of $3^{1024}$ and $3^{65536}$ are also on cards in the same format. In Table 1 the residue of $3^{2^{n}}\left(\bmod F_{m}\right)$ is described by listing its 12 least significant octal digits, and its remainder in octal modulo $2^{36}-1$ and modulo $2^{35}-1$.

Early in 1962, again partly because of available standby time, the Mersenne program, modified to check modulo $2^{35}-1$, was run for all $2^{p}-1$ for which no factor was known, with $5000<p<6000$. No primes were found. As in Hurwitz [1], the five least significant octal digits of $S_{p-1}$ are listed in Table 2.

The results for $p<3300$, mentioned by Hurwitz [1], have all been checked against the corresponding SWAC [5] and BESK [4] results. Reruns confirmed four 
7090 errors, four BESK errors (2957, 2969, 3049 and 3109), and an incorrect SWAC result for 1889. The SWAC (October 1962) confirmed the 7090 residue.

UCLA Computing Facility

University of California, Los Angeles

1. Alexander Hurwitz, "New Mersenne primes," Math. Comp., v. 16, 1962, p. 249-251.

2. A. HuRwitz \& J. L. Selfridge, "Fermat numbers and perfect numbers," Notices Amer. Math. Soc., v. 8, 1961, p. 601, abstract 587-104.

3. G. A. PAXson, "The compositeness of the thirteenth Fermat number," Math. Comp., v. 15,1961, p. 420 .

4. HaNs Riesel, "Mersenne numbers," MTAC, v. 12, 1958, p. 207-213.

5. Raphael M. Robinson, "Mersenne and Fermat numbers," Proc. Amer. Math. Soc., v. 5,1954 , p. $842-846$.

\section{Lucas' Test for Mersenne Numbers, $6000<p<7000$}

\section{By Sidney Kravitz and Murray Berg}

Alexander Hurwitz [1] reported that he had applied Lucas' test to investigate the primality of the Mersenne Numbers $M_{p}=2^{p}-1, p$ a prime, $3300<p<5000$, and discovered that $M_{4253}$ and $M_{4423}$ are prime numbers. Hurwitz [2] further states $\dagger$ that he tested all prime exponents between 5000 and 6000, where the corresponding $M_{p}$ was not known to have a factor, without discovering any new Mersenne Primes.

\begin{tabular}{cccccc}
\multicolumn{7}{c}{ TABLE } \\
\hline$p$ & $R$ & $p$ & $R$ & $p$ & $R$ \\
\hline 6007 & 07707 & 6247 & 00472 & 6659 & $75241^{*}$ \\
6037 & 21420 & 6257 & 36710 & 6661 & 27165 \\
6043 & 21605 & 6269 & 57356 & 6679 & 13275 \\
6047 & 37000 & 6299 & $71037^{*}$ & 6701 & 07636 \\
6053 & 53471 & 6329 & $25136^{*}$ & 6709 & 05700 \\
6073 & 41646 & 6337 & $21676^{*}$ & 6733 & 35544 \\
6079 & 15712 & 6359 & 51351 & 6763 & 01753 \\
6089 & 32615 & 6361 & $10027^{*}$ & 6779 & $74306^{*}$ \\
6091 & 02043 & 6451 & 23476 & 6791 & 41143 \\
6133 & 42630 & 6469 & 51252 & 6823 & $14573^{*}$ \\
6151 & 63451 & 6547 & 06546 & 6833 & 26431 \\
6211 & 71252 & 6571 & 67142 & 6857 & 63102 \\
6217 & 07377 & 6577 & $45051^{*}$ & 6907 & $46461^{*}$ \\
6221 & 24166 & 6581 & $7420^{*}$ & 6911 & 63345 \\
6229 & 06517 & 6599 & 77554 & 6971 & 65345 \\
& & & & 6991 & 50365 \\
\hline
\end{tabular}

The authors have tested the Mersenne Numbers $6000<p<7000$ without finding any new primes. A list of the five least significant octal digits of the $S_{p-1}$ th remainder from the Lucas test $\left(S_{1}=4\right)$ is given in the Table. Where a prime is missing from the list it indicates that a factor of the corresponding Mersenne Number was found by Riesel [3,4] or that an unpublished $\dagger$ factor was found by

Received February 6, 1963. Revised April 26, 1963.

$\dagger$ See pages 146, 87, and 93 of this issue of Mathematics of Computation. 